\title{
Protée
}

\section{Actualités du récit dans le champ de la linguistique des discours oraux}

\section{Le cas des narrations en situation d'entretien}

\section{Françoise Revaz et Laurent Filliettaz}

Volume 34, numéro 2-3, automne-hiver 2006

Actualités du récit. Pratiques, théories, modèles

URI : https://id.erudit.org/iderudit/014265ar

DOI : https://doi.org/10.7202/014265ar

Aller au sommaire du numéro

\section{Éditeur(s)}

Département des arts et lettres - Université du Québec à Chicoutimi

ISSN

0300-3523 (imprimé)

1708-2307 (numérique)

Découvrir la revue

Citer cet article

Revaz, F. \& Filliettaz, L. (2006). Actualités du récit dans le champ de la linguistique des discours oraux : le cas des narrations en situation d'entretien. Protée, 34(2-3), 53-66. https://doi.org/10.7202/014265ar
Résumé de l'article

Cet article aborde la problématique de l'actualité du récit d'un point de vue disciplinaire distinct - la linguistique du discours - et à propos d'un objet particulièrement énigmatique et encore mal décrit - le récit oral. Pour ce faire, il rappelle sommairement quelques éléments du modèle labovien du récit avant d'examiner, à partir d'entretiens menés avec des opératrices d'une industrie pharmaceutique, trois directions dans lesquelles ce modèle a été discuté et développé au cours des trente dernières années : la problématique de l'insertion conversationnelle des narrations ; celle de leur structuration temporelle ; et enfin celle de leur dimension évaluative. L'article contribue ainsi à mieux comprendre la nature des rapports que les approches sociolinguistiques de la narration entretiennent avec le référentiel de la narratologie structurale. 


\section{ACTUALITÉS DU RÉCIT DANS LE CHAMP DE LA LINGUISTIQUE DES DISCOURS ORAUX: LE CAS DES NARRATIONS EN SITUATION D'ENTRETIEN}

FRANÇOISE REVAZ ET LAURENT FILLIETTAZ

Au fil de son histoire, la linguistique du discours a pris l'habitude de distinguer au moins deux points de vue sur le récit (voir Laforest et Vincent, 1996). À la perspective sémiotique et narratologique, étroitement liée à l'analyse structurale des écrits littéraires et adossée à des problématiques telles que l'architecture interne des textes et leur hétérogénéité (voir notamment Adam, 1992), on oppose ainsi parfois les approches dites interactionnelles (voir Gülich et Quasthoff, 1986), qui cherchent à rendre compte de productions narratives orales issues de diverses situations de la vie quotidienne. Cette dichotomie a été abondamment commentée, que ce soit pour en relativiser les fondements ou pour en souligner les effets (voir Bres, 1994b). Elle a pourtant incontestablement ouvert la voie à des objets d'étude longtemps marginalisés comme les productions narratives orales spontanées, leur insertion dans les pratiques conversationnelles, leur développement collectivement négocié, leur indexation à des activités situées, etc.

C'est à ces objets d'étude que le présent article est consacré, ainsi qu'à une présentation de quelques développements auxquels ils ont donné lieu au cours des dernières décennies dans des champs comme la sociolinguistique, l'ethnographie de la communication, la pragmatique du discours ou encore l'analyse conversationnelle d'orientation ethnométhodologique.

Les travaux de Harvey Sacks (1992) puis ceux bien connus de William Labov (1972) ont joué, comme on le sait, un rôle déterminant dans l'émergence et le développement d'une approche interactionnelle des récits oraux. Si la narrativité ne constitue pas la préoccupation majeure de Labov ${ }^{1}$, les récits d'expérience qu'il sollicite auprès des représentants de la communauté noire américaine dans le cadre d'une enquête sociolinguistique le conduisent à explorer les fondements d'une véritable théorie du récit, qu'il exposera dans quatre articles publiés entre 1967 et 1997.

Nous rappellerons brièvement deux éléments importants de ses analyses. Le premier a trait à la distinction faite par Labov entre deux types de propositions constitutives de la narration: les propositions proprement «narratives» qui se présentent comme temporellement ordonnées et qui constituent la trame du récit, et les propositions dites «libres», à fonction d'orientation et d'évaluation, qui 
n'entrent pas dans la trame logico-temporelle du récit, mais prennent en charge sa dimension interactive. Labov a également émis l'hypothèse que le récit oral présenterait une structure particulière, dont les formes pleinement élaborées peuvent comporter les parties suivantes:

1. résumé;

2. indications;

3. développement;

4. évaluation;

5. résultat ou conclusion;

6. chute. (Labov, 1978: 298)

Dans ce modèle, non sans lien avec la narratologie structurale $^{2}$, chaque partie assume une fonction bien précise. Le résumé, situé au début du récit, permet au narrateur d'annoncer le récit qui va suivre, mais aussi de légitimer sa prise de parole et de susciter l'intérêt de ceux qui l'écoutent. Les indications jouent un rôle d' "orientation"; elles permettent de fournir des informations quant au lieu, au temps et aux acteurs impliqués dans le récit. Le développement et la conclusion constituent le noyau narratif proprement dit: il s'agit de la suite d'actions ou d'événements à rapporter. La chute, enfin, signale la fin du récit et le retour à la situation d'interlocution. L'évaluation a un statut moins clair. Tantôt Labov la considère comme une partie de la structure narrative et la cantonne donc à un endroit précis, tantôt il la définit plutôt comme une fonction qui peut apparaitre en divers endroits: "Quant à l'évaluation du récit, elle constitue une structure secondaire, concentrée dans la partie qui lui est réservée (le foyer), mais présente également sous diverses formes tout au long du récit» (ibid.: 306).

Comme le soulignent Vincent et Bres (2001), ce modèle a été critiqué à de nombreuses occasions, concernant une multitude d'aspects. Il n'a cependant jamais été rejeté complètement et semble surtout avoir joué un rôle structurant dans le développement des travaux, qui, depuis lors, s'intéressent à la modélisation des pratiques narratives orales. C'est pourquoi, plutôt que d'interroger une fois encore sa validité, nous nous appliquerons ici à identifier et à présenter trois dimensions du modèle labovien du récit qui ont fait l'objet de prolongements significatifs au cours de ces dernières décennies: l'intégration des narrations dans le processus conversationnel (partie 1); l'organisation temporelle des événements racontés (partie 2); et le fonctionnement des processus évaluatifs (partie 3).

Ces trois axes seront abordés successivement et illustrés au moyen d'une analyse de données empiriques issues d'entretiens de recherche. Dans le contexte d'un vaste programme de recherche portant sur l'analyse des actions et des discours en situation de travail $^{3}$, trois opératrices d'une usine pharmaceutique ont été filmées à leur poste de travail et interviewées. Lors des entretiens menés en tête à tête avec chacune d'elles, plusieurs thèmes sont abordés: le travail en général, le type de formation, la tâche spécifique de "remplissage manuel de poches à perfuser», les incidents et les imprévus au travail. Les entretiens sont semi-dirigés, c'est-à-dire que la chercheuse dispose d'un canevas de questions, mais laisse la place aux interviewées pour développer un thème ou raconter une anecdote. Précisons enfin que certaines des travailleuses interviewées ne sont pas francophones et qu'elles présentent à certains égards une maîtrise approximative du français.

Le corpus sélectionné comporte huit narrations qui ont toutes trait à des incidents (dysfonctionnements de machines ou accidents humains) vécus par l'interviewée en tant qu'acteur ou témoin. À ce propos, nous n'avons pas retenu les énoncés isolés qui font ponctuellement mention d'un incident comme «la semaine passée j'ai une machine qui marchait pas très bien" ou "aujourd'hui à 14 heures quand je viens il y a une dame qui m'a dit il va pas très bien». À l'image du modèle labovien, nous avons considéré comme narrative toute séquence discursive renvoyant à un événement passé et faisant l'objet d'une organisation temporelle. Ce critère minimal nous a conduits à retenir des narrations de formes et de longueurs variées, dont la majorité ne font pas l'objet d'une mise en intrigue au sens étroit du terme (voir Adam, 1992; Revaz, 1997), mais 
s'apparentent davantage à de simples comptes rendus ou relations 4 .

Lobjectif des paragraphes qui suivent est de présenter progressivement divers aspects de ces narrations et de les solliciter en regard des questions théoriques et méthodologiques qu'elles posent au modèle labovien du récit. C'est par ce moyen que nous espérons contribuer à la problématique de l'actualité du récit dans le champ de la linguistique des discours oraux.

\section{L'INSERTION DE LA NARRATION}

DANS LA DYNAMIQUE CONVERSATIONNELLE

Bien qu'orales et recueillies en situation d'entrevue, les narrations d'expériences sur lesquelles Labov a fondé sa théorie du récit se présentent de fait comme des productions langagières largement monologiques qui se prêtent mal à un traitement approfondi de la dimension proprement interactionnelle des récits. L'intervieweur se contentant de solliciter la parole du locuteur, ces productions ne permettent pas d'aborder la question de la participation active du destinataire avant, pendant et après la narration, pas plus qu'elles ne rendent possible une description des diverses modalités par lesquelles la narration s'inscrit dans l'environnement conversationnel où elle émerge.

L'analyse conversationnelle d'orientation ethnométhodologique a, de ce point de vue, apporté un prolongement bienvenu aux réflexions de Labov en mettant précisément l'accent sur les processus de coordination interactionnelle à l'œuvre au sein des événements narratifs (voir Bonu, 2001). Tel est précisément l'objectif de Jefferson (1978) qui, dans un article aujourd'hui fameux, souligne que les récits oraux sont à la fois occasionnés localement (locally occasioned) et séquentiellement déterminants (sequentially implicative) pour la suite de la conversation. Plus particulièrement, elle montre que les modalités par lesquelles les récits sont articulés à l'environnement conversationnel relèvent d'un travail essentiellement collaboratif, fondé sur une organisation séquentielle et qui n'est pas sans influence sur l'organisation interne du processus narratif ${ }^{5}$. C'est ce que nous tenterons d'illustrer cidessous au moyen de notre corpus.

Parmi les huit narrations qui composent notre corpus, sept constituent des réponses aux questions de l'intervieweuse. Elles sont donc, à l'image des récits d'expériences de Labov, implicitement sollicitées:

$\mathrm{N} 1^{6}$ : et puis sinon alors qu'est-ce qu'il y a heu est-ce qu'il y a des fois des imprévus des problèmes des incidents heu

N 2: mais des fois les machines elles ont des problèmes

N 3 : alors encore euh ah oui est-ce que: vous parliez tout à l'heure euh aussi avec vos vos collègues de des incidents: des imprévus qu'il peut y avoir ou: il y a eu ces derniers temps comme ça des des incidents techniques ou des incidents euh humains enfin

N 4: et ça arrive souvent $\uparrow$

N 6: et alors à part ces ces poches que vous avez vues que qui présentaient des défauts il y a eu d'autres: incidents: d'autres choses euh: inhabituelles: ou: à signaler

N 7: ouais (rire) qu'est-ce que: qu- il y a beaucoup de: d'accidents $\uparrow$ ou : c'est:

N 8: pas de: petits incidents: de grands incidents:

Plus précisément, les narrations d'incidents viennent souvent illustrer une réponse d'abord laconique en «oui» ou «non». Tantôt elles viennent illustrer/étayer une réponse positive:

N 1: Question: est-ce qu'il y a des fois des imprévus des problèmes des incidents heu Réponse: ah oui ça arrive aussi Illustration: comme ce matin on a eu un problème [...]

N 2: Question: mais des fois les machines elles ont des problèmes

Réponse: oui des fois les sertisseuses elles marchent pas Illustration: [...] la semaine passée je l'ai moimême j'ai une machine et puis qui marchait pas très bien $[\ldots]$ 
Tantôt elles viennent apporter une «contreillustration" à une réponse négative:

N 3: Question: il y a eu ces derniers temps comme ça des des incidents techniques ou des incidents euh humains enfin

Réponse: non

Contre-illustration: bon l'autre jour la dame bon elle a eu elle a eu une (rires) bon c'est la dame que je vous parle qui est un peu olé olé là qui qui a eu une sertisseuse qui lui est tombée dessus [...]

N 6: Question: il y a eu d'autres: incidents: d'autres choses euh: inhabituelles: ou: à signaler Réponse: oh NON $\uparrow$ Contre-illustration: il y en a des: des fois euh il y en a des PLUS de points noirs que on trouve [...]

N 8: Question: pas de: petits incidents: de grands incidents:

Réponse: non

Contre-illustration: il y a juste une fois: mais c'était avant peut-être euh même DEUX mois trois mois il y a un monsieur qui a tombé juste là dans les:.. entre les nutritions et puis les bureaux [...]

Une des huit narrations est moins facilement localisable. Il s'agit de la narration 4 (mise en évidence ci-dessous dans la section grisée) qui n'arrive qu'au terme de plusieurs questions de relance de l'intervieweuse $(F R)$ :

FR: et ça arrive souvent $\uparrow$

$M^{m e} P$ : non ça va maintenant mais au début

FR: $\quad$ XXX la lettre de Monsieur B qui dit qu'il y a souvent des accidents [ouais] quand même

$\mathrm{M}^{m e} P$ : ben ouais peut-être dans les autres zones mais au début ici c'était L'HORREUR hein. hein [ouais $\uparrow$ ] parce que c'était toujours mouillé: les gens ils ils avaient pas conscience que quand ils mouillaient fallait nettoyer:

FR: mais quand vous dites au début quand [e-] il y a: $\mathrm{M}^{\text {me }} P$ : au début il y a. pas euh $\mathrm{f}$ - ben surtout au début aussi parce que $\mathrm{c}$ - les tuyaux maintenant c'étaient pas les mêmes tuyaux qu'il y a. que il y a maintenant. plus euh:. euh les gens s-ils ils ils étaient pas conscients que fallait passer la la perserpillière ou passer l'aspirateur quand c'était mouillé maintenant ça va $\downarrow$. mieux maintenant $\downarrow$ bon c'est

FR: $\quad$ mais quand c'était au début il y a une année il y a dix ans

$M^{m e} P$ : oh non il y a cinq six ans il y a dix ans oui il y a. il y a ça il y a il y a ouais il y a quatre cinq ans peut-être ça va MIEUX. parce que c'était TOUjOURS mouillé un temps ici tout le temps et puis maintenant ça va $\downarrow[\mathrm{mm}]$ hein. et c'est bon maintenant. hein alors BON au début il y a eu les MACHINES là les nouvelles machines il y a beaucoup de: d'HOMMES qu'ont eu des des qui ont été blessés $\downarrow$ maintenant ça va mieux maintenant [mm] hein bon il y a un monsieur qui est là qui a eu la MAIN arrachée là il a été très longtemps parce qu'il fallait lui regreffer de la peau: sur la main et tout hein. [ah ouais $\uparrow$ ] ouais ouais OUAIS il y a eu beaucoup de d'hommes qui ont eu des problèmes UN JOUR j'étais: j'étais pour aller à la pause j'entendais crier puis il y avait un ho- un homme que qui s'est CASSÉ le bras il avait pris la main dans une la main dans iparce qu'il a il a au lieu d'arrêter la machine il a pas arrêté la machine il a mis la main où il fallait pas et puis bon il s'est cassé le bras [mm] hein parce que: il devait cette machine là il devait pas l'ar- l- l-s'il l'arrêtait fallait encore attendre UN QUART D'HEURE pour qu'elle redémarre il aurait mieux valu parce qu'il aurait pas eu la la jam- le bras cassé $\uparrow$ hein

À la question de savoir si les accidents sont fréquents ("et ça arrive souvent $\uparrow$ »), $\mathrm{M}^{\mathrm{me}} \mathrm{P}$ répond négativement tout en mentionnant qu'avant la situation était différente: «non ça va maintenant mais au début" (sous-entendu: "oui il y avait souvent des accidents"). Devant l'insistance de l'intervieweuse qui vient de lire une lettre adressée à toutes les ouvrières signalant une recrudescence d'accidents, $\mathrm{M}^{\text {me }} \mathrm{P}$ reconnaît que «ouais peut-être» il y a des accidents, mais elle nuance tout de suite en disant que cela doit 
arriver dans les autres zones de production. Elle reconnaît toutefois que dans sa zone «au début c'était L'HORREUR parce que c'était toujours mouillé», déclaration dont il faut déduire qu'il y avait des accidents parce que les gens glissaient sur le sol mouillé. $\mathrm{M}^{\text {me }} \mathrm{P}$ va répéter cinq fois des variantes de «maintenant ça va mieux». À l'avant-dernière occurrence ("c'est bon maintenant»), elle cite une autre cause d'accidents dans le passé, à savoir la présence de nouvelles machines ( BON au début il y a eu les MACHINES là les nouvelles machines») et mentionne des cas d'accidents ( $i$ il y a beaucoup de: d'HOMmes qu'ont eu des des qui ont été blessés $\downarrow$ "). À la dernière occurrence de "ça va mieux maintenant", elle concède toutefois qu' «il y a un monsieur qui est là qui a eu la MAIN arrachée là". La réaction étonnée de l'intervieweuse ("ah ouais $\uparrow$ ») semble déclencher d'autres souvenirs d'accidents («ouais ouais OUAIS il y a eu beaucoup de d'hommes qui ont eu des problèmes») jusqu'à lui faire raconter, plus longuement cette fois, un accident. On voit bien ici comment se mettent en place progressivement les conditions d'une énonciation collectivement négociée de la narration.

Dans notre corpus, une seule narration (N5) ne constitue pas une réponse à une question de l'intervieweuse. Elle apparaît spontanément comme un exemple d'incident à l'intérieur d'une séquence englobante où l'opératrice interviewée relate pas à pas le détail de ses activités habituelles (voir Revaz, 2004): $\mathrm{M}^{\text {me }} \mathrm{D}:[\ldots]$ et puis PLUS TARD. peut-être un petit peu avant la pause vers cinq heures et demie. moi je me lève et puis j'ai coupé cette cinq pièces et puis j'ai M'AGRAFÉ avec ce ticket-t. [mm] de là là il était sûr de ça vient cette poche que ça vient dans cette bac-là $\downarrow$ et puis c'est moi que j'ai signé j'ai contrôlé et puis là on est sûr que c'est moimême que j'ai fait $\downarrow$ j'ai donné aux dames et puis quand: vous avez vu quand je viens dans ligne il y a cinq poches qui a traîné à côté $[\mathrm{mm}]$ moi je me demandais qu'est-ce que c'est ça $\uparrow[\mathrm{m}]$. parce que ça m'intéresse tout de suite il y a PAS de feuille en haut et PAS de fiche qui soit ou même ouvrir XX poche et puis j'ai regardé j'ai demandé aux dames et puis elle m'a dit il y a un point noir il y en a d'écran qui était:. il était pas bon on peut pas laisser passer parce que il est pas visible $[\mathrm{mm}]$ et puis moi j'ai mis avec un crayon: noir comme ça on va montrer au chef [mm] et puis on fait-t-encore les: qui fait plus attention au formage [voilà] comme ils ont fait

Quant aux clôtures des narrations, elles peuvent s'effectuer selon différentes modalités. Parfois, elles consistent en une reprise de l'énoncé introductif par lequel l'opératrice a admis qu'un accident est arrivé, avec toutefois cette précision que ce n'est pas la règle générale. Elles concluent alors le mouvement argumentatif amorcé en début de tour de parole et font ainsi explicitement référence aux conditions dialogiques dans lesquelles elles ont été introduites:

N 7: Introduction: dernières temps il y a une dame qu'il a un sertisseuse qui a tombé sur sur elle sur les pieds

Clôture: [...] mais il tombe normalement. par rapport $\mathrm{X}$ il tombe pas du tout hein

N 8: Introduction: il y a juste une fois: mais c'était avant peut-être euh même DEUX mois trois mois il y a un monsieur qui a tombé juste là dans les:.. entre des nutritions et puis les bureaux Clôture: [...] ça arrive mais c'est pas souvent $\downarrow$

N 1: Introduction: ah oui ça arrive Clôture: [...] ça c'est rare que ça arrive ça

Ces effets de boucle argumentative ne sont cependant pas toujours attestés. Ainsi, dans un certain nombre de cas, la narration se clôt simplement sur la mention du dernier événement ou de la dernière action de la chaîne narrative:

N 2: Clôture: [...] et puis là ça risque plus rien que ça vient de qu'il soit pas bon de volume ça corresponde pas les grammes et tout ça

N 6: Clôture: [...] et puis j'ai laissé à côté pour euh: agrafer avec le: ticket

N 5: Clôture: [...] et puis moi j'ai mis avec un crayon: noir comme ça on va montrer au chef [mm] et 
puis on fait-t-encore les: qui fait plus attention au formage [voilà] comme ils ont fait

Enfin, il peut arriver que la clôture soit amenée par le biais d'une évaluation finale prenant la forme d'une morale, mais qui ne fait pas explicitement référence au contexte dialogique dans lequel la narration a été initiée:

N 3: Clôture: [...] bon ça a pas arrêté trop la ligne mais bon je s-moi je me suis occupée j'ai perdu quarante-cinq minutes hein $\downarrow$ fallait bien s'en occuper quoi

N 4: Clôture: [...] cette machine là il devait pas l'ar- 1-1s'il l'arrêtait fallait encore attendre UN QUART D'HEURE pour qu'elle redémarre il aurait mieux valu parce qu'il aurait pas eu la la jam- le bras cassé $\uparrow$ hein

\section{LA RÉORGANISATION NARRATIVE DES ÉVÉNEMENTS PASSÉS}

Dans l'acception que nous en donnons ici, toute narration renvoie nécessairement à une suite événementielle temporellement ordonnée. Pour Labov, cette condition constitue explicitement le critère définitoire central de la narrativité, le récit étant envisagé comme

[...] une méthode de récapitulation de l'expérience passée consistant à faire correspondre à une suite d'événements (supposés) réels une suite identique de propositions verbales [...] si bien que l'inversion de cet ordre entraîne une modification de l'enchaînement des faits reconstitué au plan de l'interprétation sémantique. (Labov, 1978 : 295-296)

Ainsi, un isomorphisme semble ici posé entre l'enchaînement des propositions langagières et l'ordre temporel des événements représentés.

Cette hypothèse forte d'une isochronie entre les plans textuel et référentiel a donné lieu elle aussi à d'abondants commentaires, qui semblent se cristalliser autour de deux points majeurs. Le premier consiste à remettre en question, avec Ricœur, l'idée selon laquelle la temporalité des événements pourrait s'envisager indépendamment des processus narratifs qui s'y rapportent. C'est du moins ce que soutient Bres, lorsqu'il montre qu'il y a lieu d'inverser le rapport hiérarchique classiquement perçu entre le plan textuel et le plan référentiel:

Le réel est inépuisable, infini: il n'est d'histoire que pour/par le sujet. Dans le réel, les événements sont légion qui surviennent à la ligne du temps. Le récit met ordre et relief: il sélectionne et hiérarchise (à l'aide notamment des temps verbaux). La successivité n'est donc pas donnée (passivement) au récit par l'événement mais construite (activement) par la mise en intrigue.

(Bres, 1994a: 75)

Le second point de controverse concerne l'idée même d'une jonction temporelle nécessairement ascendante pour le cas de la narration. Si la textualité narrative semble en effet progresser temporellement, il faut bien reconnaître que l'ordre des propositions textuelles n'est pas toujours dans un rapport d'homologie avec l'ordre chronologique des événements racontés. Outre des relations de progression, des rapports de régression ou de simultanéité sont également attestés sur le plan empirique, comme le montre bien Bres:

Il apparaît à l'analyse que la relation de progression non inclusive, si elle structure très majoritairement l'enchaînement des propositions de la textualité narrative $d u$ récit conversationnel, n'est cependant pas la seule: on trouve également, certes très peu fréquemment, la simultanéité, la régression, l'inclusion, la composition. Dès lors, la position que défend Labov (à partir de 1972) selon laquelle le "narrative sequencing» et lui seul régirait les enchaînements propositionnels de la textualité narrative se trouve mise en débat. (2001: 44)

Ainsi, dans les narrations orales de notre corpus, on observe des modalités variées par lesquelles la narration organise les événements passés: progressive ou régressive avec le plus souvent un brouillage dans la chaîne chronologique dû à la présence d'événements anticipés ou répétés. Parfois, localement, le temps n'avance pas et une pluralité de propositions peut renvoyer à des événements simultanés.

N 2: $\left(\mathrm{M}^{\mathrm{me}} \mathrm{D}\right)^{7}$ :

1. moi je vous explique un petit peu comme ça 
2. moi je mettais par exemple pour $1000 \mathrm{ml}$ sur l'acide aminé

3. moi je mettais $1400[\mathrm{~mm}]$ pour que ça pèse dans les poches 434 les grammes [mm]

4. et puis chez moi ça

5. moi je mettais 1400

6. mais après je mettais jusqu'à 1500

7. ça fait 100 grammes de différent

8. et puis c'est pas juste

9. là je vois tout de suite que ça marche pas

10. il y a quelque chose que ça va pas [mm]

11. et puis je prenais tout de suite dix poches une après l'autre tout de suite

12. et j'ai pesé

13. et chacune qui était différent

14. et puis là j'ai appelé le mécano mécanicien [mm]

15. et puis j'ai dit monsieur s'il vous plaît tu regardes parce que il y a quelque chose qui va pas

16. et puis moi j'arrête

17. et dans les papiers je marque j'ai arrêté à: par exemple à huit heures moins quart jusqu'à la machine qui était qui est prête

18. et puis après

19. je sais pas comment elle s'appelle

20. si on a besoin changer quelque chose dans les machines après on a besoin faire un petit rinçage de dix mille [mm]

21. et puis après ça on fait encore deux poches pour jeter

22. et puis après on remplit pour les pour nous [d'accord] pour qu'ils soient bien les sacs

23. et puis là ça risque plus rien que ça vient de qu'il soit pas bon de volume ça corresponde pas les grammes et tout ça

La narration de $\mathrm{M}^{\text {me }} \mathrm{D}$ porte sur un problème technique rencontré récemment sur son poste de travail. Cette narration se présente comme globalement progressive, c'est-à-dire que la suite des clauses narratives renvoie à une progression chronologique des actions de la narratrice. Cette progression est marquée par les organisateurs temporels APRÈS, ET PUIS, ET PUIS LÀ, etc. Pourtant, si globalement la narration progresse, chaque nouvelle clause ne fait pas pour autant avancer le temps. Au début de la séquence, par exemple, $\mathrm{M}^{\mathrm{me}} \mathrm{D}$ reste focalisée sur la première action, à savoir le réglage de sa machine sur $1400 \mathrm{ml}$ (clauses 2 à 5). Entre ces clauses, il n'y a certes pas de progression temporelle des événements, mais l'information progresse malgré tout puisque l'on apprend d'abord qu'il s'agit d'une poche de $1000 \mathrm{ml}$ d'acides aminés (clause 2), puis la raison pour laquelle $M^{\text {me }} \mathrm{D}$ règle sa balance à 1400 (clause 3). À la clause 5, la narratrice reformule la première action («moi je mettais $1400 »)$, ce qui lui permet d'enchaîner sur l'action suivante (clause 6 : «mais après je mettais jusqu'à 1500»). Les clauses 7 à 10 ne sont pas narratives au sens technique du terme $^{8}$. En effet, $\mathrm{M}^{\mathrm{me}} \mathrm{D}$ y commente la deuxième action. Il en est de même aux clauses 13, 19 et 23. La trame narrative semble donc constituée ici des clauses 2 à $6,11,12,14$ à 18 et 20 à 22.

À d'autres moments, comme dans la narration 8, des mouvements régressifs sont également attestés, qui entrent dans des combinaisons complexes avec l'organisation chronologique des événements racontés:

N 8: $\left(M^{m e} \mathrm{D}\right)$ :

1. non il y a juste une fois:

2. mais c'était avant

3. peut-être euh même DEUX mois trois mois

4. il y a un monsieur qui a tombé juste là dans les:.. entre des nutritions et puis les bureaux

5. parce que il y a un petit peu mouillé le parterre

6. et puis lui i-il était: allé vite [mm]

7. il a glissé

8. et puis il a tombé

9. il a cassé arcades là [...]

10. et puis il vient ici au bureau

11. et: le sang qui a tout coulé

12. j'ai mis un peu désinfecté [mm]

13. et puis j'ai mis une poudre blanche.

14. je sais pas comment elle s'appelle en français mais on a des des médicaments pour tout de suite euh. donner un petit aide première $[\mathrm{mm}]$ 
15. puis on a mis sparadrap

16. et puis il est parti à l'hôpital.

17. là ils ont mis: quatre ou six points

18. ils ont coudré $\downarrow$ [ouais]

Cette narration commence in media res avec la mention de l'événement central «il y a un monsieur qui a tombé juste là dans les:.. entre des nutritions et puis les bureaux» (clause 4). La clause qui suit remonte, dans un mouvement régressif, à la cause initiale de la chute: "parce que il y a un petit peu mouillé le parterre» (clause 5). Ensuite, dans un mouvement progressif, l'enchaînement logico-causal est présenté: "et puis lui i- il était: allé vite» (clause 6), «il a glissé» (clause 7), « et puisil a tombé» (clause 8). La clause 8 clôt la première partie événementielle en répétant l'information donnée d'entrée. Par la suite, la progression du temps racontant recoupe la progression du temps raconté, à savoir la blessure et les soins qui ont suivi, d'abord sur place, puis à l'hôpital.

On constate enfin que le "et puis" de la clause 6 signale davantage une progression du temps racontant que celle du temps raconté, le plus-que-parfait marquant très clairement le mouvement rétrospectif («il était allé vite», clause 6). On touche ici à la question complexe des mécanismes énonciatifs à l'œuvre dans la narration orale, question que nous développerons davantage dans les paragraphes suivants.

\section{3. ÉVALUATIONS ET COMMENTAIRES:}

LA DIMENSION ÉNONCIATIVE DES PROCESSUS NARRATIFS

Si toute narration est identifiable par la présence d'une trame temporelle qui renvoie à une chronologie événementielle, il est cependant rare qu'elle se réduise à cette dernière. En général, une narration est composée d'énoncés qui, s'ils ne font pas proprement avancer le temps, apportent des éléments descriptifs, des jugements de valeur, des explications et des commentaires, toutes sortes de compléments d'information qui contribuent à l'interprétation par l'interlocuteur des événements rapportés. Outre les éléments de résumé et d'indication, qui, dans le modèle labovien du récit, sont matérialisés par ces propositions libres, c'est principalement autour du concept d'évaluation que s'est cristallisée, dans la tradition linguistique, une réflexion portant sur la prise en charge par le narrateur des événements racontés. C'est du moins le mérite que lui reconnaît un auteur comme Bres:

La notion d'évaluation, si floue soit-elle parfois chez Labov à force d'extension, a l'immense mérite de placer le récit dans l'interaction verbale, d'en faire un acte de langage dont la structure interne est façonnée par l'interlocution. Le récit est toujours récit pour l'autre; il est quête de l'assentiment de l'autre. L'intention du narrateur n'est pas simple intention de communiquer une chronologie événementielle: c'est aussi (d'abord?) l'intention de produire un effet. (1994a: 83-84)

On rappellera cependant que cet «effet» que vise à produire le narrateur et que prend en charge le concept d'évaluation s'avère en réalité étroitement dépendant des spécificités des données que sont les récits de bagarres ou de dangers de mort sollicités par Labov. Plus précisément, il découle, comme l'a bien montré Bonu (2001: 53), de la nécessité dans laquelle se trouve le narrateur de justifier le caractère extraordinaire - racontable - de l'épisode narré et ainsi d'éviter le spectre d'une réaction telle que «et alors?» énoncée par l'interlocuteur. Dans cette perspective, le concept d'évaluation défini par Labov désigne l'ensemble des moyens mobilisés par le narrateur «pour indiquer le propos de l'histoire, sa raison d'être: pourquoi il la raconte, où il veut en venir» (1978: 301).

Pourtant, comme l'ont bien montré les travaux de Vincent (1996), le critère de racontabilité ne s'applique de loin pas littéralement à l'ensemble des pratiques narratives attestées. Aussi importe-t-il, selon nous, de donner à la problématique de l'évaluation une portée plus large, qui ne se limite pas à la mise en évidence de ce qui rend les événements racontés mémorables, mais qui recoupe plus généralement l'ensemble des procédés par lesquels le narrateur gère la prise en charge énonciative de son récit: comment il se montre ou au contraire s'efface; comment il 
endosse les propos tenus ou au contraire s'en distancie, etc.

Dans notre corpus, ces procédés évaluatifs reposent sur une vaste palette de moyens linguistiques que nous présenterons sommairement avant de les combiner dans une analyse plus détaillée de deux narrations: des commentaires métadiscursifs et métalinguistiques; des commentaires explicatifs; des expressions modales; des expressions axiologiques.

a) Commentaires métadiscursifs et métalinguistiques

Certains énoncés consistent en un commentaire du narrateur sur son discours, sur sa manière de parler (métadiscursif) ou sa façon de nommer les choses (métalinguistique). Ils constituent un premier type de trace laissée par l'énonciateur dans sa narration:

- je vous explique un petit peu (N2)

- je parle un peu vite (N3)

- je sais pas comment elle s'appelle (N2)

- je sais pas comment elle s'appelle en français (N8)

- moi j'appelle projet TOP (N3)

\section{b) Commentaires explicatifs}

Une seconde catégorie de marquage énonciatif réside dans ce que nous considérons ici comme des commentaires explicatifs. Dans nos données, ces commentaires sont de trois types. Soit ils explicitent "dans quel but» quelque chose est fait, soit ils en donnent la "raison", le "motif", soit encore ils présentent «sous quelle condition» on peut/doit le faire. Dans le premier cas, ils sont introduits par la marque linguistique POUR, suivie d'une proposition infinitive ou complétive; dans le deuxième cas, par la marque PARCE QUE, suivie d'une complétive; dans le troisième enfin, par la marque SI.

Les énoncés introduits par POUR ouvrent une perspective dans le déroulement de l'action représentée. Ils décrivent en même temps un état à venir et un but:

- je mettais $1400[\mathrm{~mm}]$ pour que ça pèse dans les poches 434 les grammes $(\mathrm{N} 2)$

- et puis après on remplit pour les pour nous [d'accord] pour qu'ils soient bien les sacs (N2)

- j'ai laissé à côté pour euh: agrafer avec le: ticket-t (N6)

Les commentaires introduits par PARCE QUE et se rapportant aux motifs ou raisons d'agir sont beaucoup plus nombreux. Nous n'en citerons que quelques-uns:

- on a mis à part derrière parce qu'on savait pas si ça allait passer (N1)

- j'ai mis le poche à côté [voilà c'est ça] parce que ça on peut pas remplir (N6)

- ça arrive mais c'est pas souvent $\downarrow[\mathrm{mm}]$ pas souvent parce que quand même on fait-tattention (N8)

- on a rincé un peu les machines on a pris les tuyaux pour rincer quand même pour que parce que dans les tuyaux il reste encore de la solution d'avant (N1)

Enfin, plusieurs commentaires sont introduits par SI:

- si on a besoin changer quelque chose dans les machines après on a besoin faire un petit rinçage de dix mille $[\mathrm{mm}]$ et puis après ça on fait encore deux poches pour jeter et puis après on remplit pour les pour nous (N2)

- s'il l'arrêtait fallait encore attendre UN QUART D'HEURE pour qu'elle redémarre (N4)

- si je vois quelqu'un qui pouvait faire acc-une accident on dit toujours eh faites attention là ça pouvait être une accident (N8)

- si ça commence changer les volumes il faut tout de suite arrêter il faut pas dire ah peut-être il va mieux aller ou ça va y aller comme ça parce que ça va pas du tout (N2)

La fonction de ces commentaires en SI réside dans la mention d'une autre possibilité d'agir, non réalisée dans la trame événementielle de la narration en cours. À l'image des autres commentaires explicatifs identifiés ci-dessus, ils soulignent l'effort que déploient les narratrices pour rendre les actions représentées interprétables par la chercheuse.

c) Les expressions modales

Un autre procédé réside dans le marquage modal des éléments de la narration. Les narratrices recourent 
ainsi parfois à des modalisations épistémiques, par lesquelles elles se positionnent, en termes de doute ou de certitude à l'égard des contenus assertés:

- je sais pas combien on a fait j'en sais rien moi j'ai pas regardé le compteur (N1)

- là je vois tout de suite que ça marche pas il y a quelque chose que ça va pas (N2)

- je sais pas [...] je sais pas comment elle a fait (N7)

Dans ces exemples, les verbes SAVOIR ou VOIR traduisent la perception ou la capacité du narrateur. Ils véhiculent ainsi des éléments de représentation de l'énonciateur dans son propre discours.

\section{d) Les expressions axiologiques}

Enfin, on notera, à certaines reprises, que les narratrices énoncent des jugements de valeur à l'égard des éléments représentés dans leurs narrations. Elles recourent ainsi à des expressions axiologiques, par lesquelles s'exprime linguistiquement la subjectivité de l'énonciateur (voir Kerbrat-Orecchioni, 1980):

- il aurait mieux valu (N4)

- c'est la dame que je vous parle qui est un peu olé olé là (N3)

- $\quad$ on a eu un problème (N1)

- $\quad$ parce que ça va pas du tout (N2)

Pour compléter ce bref aperçu des procédés évaluatifs, nous terminerons par l'analyse de deux narrations (N3 et N7), qui, d'une part, présentent de nombreuses marques de prise en charge énonciative et, d'autre part, renvoient au même accident, raconté par deux narratrices différentes.

N3: M $^{\text {me }} P$

$M^{m e} P$ : non bon l'autre jour la dame bon elle a eu elle a eu une (rires) bon c'est la dame que je vous parle qui est un peu olé olé là qui qui a eu une sertisseuse qui lui est tombée dessus mais elle l'a cherch- elle CHERCHE c-bon je veux pas l- lui trouver des excuses de rien du tout mais elle était elle agit TELLEMENT alors vous savez il y a des $\mathrm{f}$-bon on a parlé au à $\mathrm{l}$ - au au au projet TOP ça on va rectifier tout ça il y a beaucoup de tuyaux qui passent qui passent

FR: c'est quoi le G TOP

$M^{m e} P$ : heu le projet TOP là [rires] le le là c'est le oui moi j'appelle projet TOP je parle un peu vite

FR: $\quad$ ah non non non ça va

$M^{\text {me }} P$ : euh :. la euh la dame est souvent elle à s- elle est elle travaille debout elle a sa chaise puis elle bouge toujours toujours et puis euh elle a ar-on a arrêté et puis elle s'est prise dans les fils de la sertisseuse qui était derrière je sais pas comment elle a fait puis elle lui est tombée dessus alors bon moi euh bon ça a pas arrêté trop la ligne mais bon je s- moi je me suis occupée j'ai perdu quarante-cinq minutes hein_fallait bien s'en occuper quoi

N7: $\mathrm{M}^{\text {me }} \mathrm{D}$

$M^{\text {me }} D$ : bon parce que dernières temps il y a une dame qu'il a un sertisseuse qui a tombé sur sur elle sur les pieds et puis c'était dangereux parce que les machines ils sont trop serrés et puis il y en a des fils par terre. et puis on a besoin beaucoup de fils à cause de l'air à cause de: remplissage il y a partout des tuyaux. et puis elle euh je sais pas elle était attachée un peu avec le chaise et:. je sais pas comment elle a fait et puis cette nouveau sertisseuse qu'est-ce qu'on a ils sont pas très stables [mm]. ils sont préparées mais nous on a on a demandé au chef s'il pouvait nous faire quelque chose qui était PLUS lourd sur les tables comme ça ils tombent pas facilement

FR: $\quad$ parce que

$\mathrm{M}^{\text {me }} \mathrm{D}$ : et puis

FR: $\quad$ parce qu'elles tombent souvent $\uparrow$

$M^{\text {me }} D: \underline{\text { euh }}$

FR: $\quad$ il y a souvent des accidents $\uparrow$

$M^{\text {me }} D$ : parce que cette dame euh c'était $M^{\text {me }} M$ il était il est un peu pressé parce qu'il voulait faire son quantité et puis il s'est dépêché et puis là il était attaché un peu avec les fils et puis il a tombé très facilement $\downarrow$. mais il tombe normalement. par rapport $\mathrm{X}$ il tombe pas du tout hein [ah bon] parce que ça c'est une machine qu'il faut pas 
tomber par terre (rire). c'est:. pour utiliser tous les jours et puis:.[mm]. il faut pas qu'il tombe il faut pas:. il faut qu'il être toujours à sa place

Comme on peut le constater, les deux narrations relatent le même accident:

Une opératrice s'est prise dans les fils d'une sertisseuse $\rightarrow$ la sertisseuse lui est tombée dessus.

Toutes deux débutent par un résumé: $\mathrm{M}^{\mathrm{me}} \mathrm{P}(\mathrm{N} 3)$ : bon l'autre jour la dame bon elle a eu elle a eu une (rires) [...] une sertisseuse qui lui est tombée dessus; $\mathrm{M}^{\mathrm{me}} \mathrm{D}(\mathrm{N} 7)$ : dernières temps il y a une dame qu'il a un sertisseuse qui a tombé sur sur elle sur les pieds

et présentent une structure événementielle minimale composée de trois ou quatre clauses narratives:

$M^{\text {me }} P(\mathrm{~N} 3)$ :

1. elle a ar- on a arrêté

2. et puis elle s'est prise dans les fils de la sertisseuse qui était derrière

3. puis elle lui est tombée dessus

$\mathrm{M}^{\text {me }} \mathrm{D}(\mathrm{N} 7)$ :

1. elle était attachée un peu avec le chaise

2. et puis il s'est dépêché

3. et puis là il était attaché un peu avec les fils

4. et puis il a tombé très facilement $\downarrow$.

Pourtant, si les trames narratives se présentent comme peu développées, les procédés évaluatifs qui s'y rapportent occupent quant à eux une place prépondérante. On retrouve, par exemple dans ces narrations, certains des procédés linguistiques décrits plus haut: des commentaires explicatifs en PARCE QUE ("parce que les machines ils sont trop serrés»), des expressions modales ( je sais pas comment elle a fait»), des expressions axiologiques dénotant une position appréciative ( $«$ mais elle l'a cherch- elle CHERCHE», «je veux pas l- lui trouver des excuses», "c'était dangereux", etc.).

Mais, plutôt que de passer par un relevé exhaustif de tous les énoncés évaluatifs, il semble plus intéressant de mettre en évidence les éléments sur lesquels ils portent. Ce qui frappe en effet dans la mise en regard de ces deux narrations, c'est que si la trame événementielle se présente comme identique, les narratrices ne commentent pas du tout les mêmes choses. La narration de $\mathrm{M}^{\mathrm{me}} \mathrm{P}(\mathrm{N} 3)$ donne à voir un accident dont la responsabilité incombe totalement à un agent humain ("la dame qui est un peu olé olé»), alors que la narration de $\mathrm{M}^{\mathrm{me}} \mathrm{D}(\mathrm{N} 7)$ insiste plus sur les causes matérielles («les machines trop serrées» et «les fils par terre»). Certes, les narratrices reconnaissent toutes deux ne pas savoir comment $\mathrm{M}^{\mathrm{me}} \mathrm{M}$ a fait pour se blesser, mais leurs points de vue sur l'accident diffèrent comme le montre la nature même des commentaires et des évaluations. Par exemple, $\mathrm{M}^{\mathrm{me}} \mathrm{P}$ ne cesse de juger $\mathrm{M}^{\mathrm{me}} \mathrm{M}$ :

- c'est la dame que je vous parle qui est un peu olé olé

- mais elle l'a cherch- elle CHERCHE

- mais elle était elle agit TELLEMENT alors vous savez il y a des $\mathrm{f}$ -

- la dame est souvent elle à s- elle est elle travaille debout elle a sa chaise puis elle bouge toujours toujours

Énoncés dépréciatifs et accents d'insistance concourent à brosser le portrait d'une personne à qui incombe toute la responsabilité de l'accident. Le problème du matériel ( $«$ il y a beaucoup de tuyaux qui passent qui passent») est évoqué rapidement mais, pour un auditeur non averti, la relation entre la présence de tuyaux et l'accident n'est pas évidente.

À l'inverse, $\mathrm{M}^{\mathrm{me}} \mathrm{D}$ insiste sur l'enchaînement causal qui a conduit à l'accident:

- c'était dangereux parce que les machines ils sont trop serrés et puis il y en a des fils par terre. et puis on a besoin beaucoup de fils à cause de l'air à cause de: remplissage il y a partout des tuyaux

- cette nouveau sertisseuse qu'est-ce qu'on a ils sont pas très stables

Même lorsqu'elle parle de sa collègue, $\mathrm{M}^{\mathrm{me}} \mathrm{D}$ tente d'expliquer l'accident sans déprécier pour autant son comportement: 
- $\quad$ parce que cette dame [...] il était il est un peu pressé parce qu'il voulait faire son quantité

L'empressement de $\mathrm{M}^{\mathrm{me}} \mathrm{M}$ est linguistiquement atténué ("un peu pressé») et ses raisons d'agir sont présentées comme louables. En effet, «vouloir faire son quantité» n'est pas un mobile égoïste mais profite aussi aux collègues dans la mesure où chaque équipe est gratifiée d'un bonus selon la quantité de poches produites.

Les narrations se terminent toutes deux par une chute évaluative:

$\mathrm{M}^{\text {me }} \mathrm{P}$ : alors bon moi euh bon ça a pas arrêté trop la ligne mais bon je s- moi je me suis occupée j'ai perdu quarante-cinq minutes hein $\downarrow$ fallait bien s'en occuper quoi

$\mathrm{M}^{\text {me }} \mathrm{D}$ :mais il tombe normalement. par rapport $\mathrm{X}$ il tombe pas du tout hein [ah bon] parce que ça c'est une machine qu'il faut pas tomber par terre (rire). c'est: . pour utiliser tous les jours et puis:.[mm]. il faut pas qu'il tombe il faut pas:. il faut qu'il être toujours à sa place

$\mathrm{M}^{\text {me }} \mathrm{P}$ relève les désagréments causés par l'accident: arrêt (même minime) de la ligne et perte de temps pour elle. Elle conclut par une morale: «fallait bien s'en occuper quoi». $M^{\text {me }} \mathrm{D}$, pour sa part, termine sa narration sur le problème des machines en répétant cette prescription «il ne faut pas qu'elle tombe», «il faut qu'elle reste à sa place», qui fait écho à la demande adressée au chef ("on a demandé au chef s'il pouvait nous faire quelque chose qui était PLUS lourd sur les tables comme ça ils tombent pas facilement»).

On constate donc que les énoncés qui accompagnent la trame événementielle sont ici prépondérants. S'ils jouent un rôle majeur dans l'interprétation narrative, c'est non seulement en ce qu'ils véhiculent des éléments d'information permettant au narrataire d'accéder à une compréhension fine des événements relatés, mais c'est aussi en ce qu'ils constituent pour les narratrices une ressource déterminante pour se mettre en scène et négocier des traits identitaires. On notera que, de ce point de vue, $\mathrm{M}^{\mathrm{me}} \mathrm{D}$ et $\mathrm{M}^{\mathrm{me}} \mathrm{P}$ endossent des postures remarquablement différentes dans leur discours: si $\mathrm{M}^{\mathrm{me}} \mathrm{D}$ se positionne comme une employée soucieuse de la sécurité de l'infrastructure ("c'était dangereux", «ils sont pas très stables", "il faut pas qu'il tombe») et subordonnée à son supérieur hiérarchique («nous on a on a demandé au chef»), $\mathrm{M}^{\mathrm{me}} \mathrm{P}$ assume en revanche une position bien plus évaluative ("c'est la dame que je vous parle qui est un peu olé olé», «elle CHERCHE») et marque ainsi un rapport d'autorité envers ses collègues. En définitive, c'est donc la délicate question de la présentation de soi et de la préservation des faces (Goffman, 1959) que véhiculent les narrations orales et plus particulièrement les procédés évaluatifs qui leur sont associés 9 .

\section{REMARQUES CONCLUSIVES}

Dans cette contribution, nous avons cherché à aborder la problématique de l'actualité du récit du point de vue d'un champ disciplinaire précis - la linguistique du discours - et à propos d'un objet particulièrement énigmatique et encore mal décrit - le récit oral.

Évidemment, notre parcours ne rend pas justice à la richesse des réflexions conduites par les linguistes en écho aux travaux de Labov. D'autres axes auraient ainsi pu être abordés, comme la problématique de l'hétérogénéité énonciative des productions narratives orales et la présence du discours rapporté, la question des fonctions situationnelles de la narration (voir Charaudeau, 1994; Filliettaz, 2001, 2002) ou encore celle des différentes modalités de textualisation de l'action (voir Revaz, 1997).

Cette étude aura permis de mettre en évidence à la fois les continuités et les déplacements opérés par le champ de la sociolinguistique de l'interaction à l'égard de la narratologie structurale. Continuités en ce que la structure événementielle reste perçue comme un élément organisationnel définitoire de la narrativité. Déplacements en ce que cette trame narrative ne peut être étudiée qu'en référence aux activités langagières 
qui la supportent et lui donnent sens. C'est en définitive dans cette mise en regard des ressources sémiotiques avec les pratiques sociales que résident à nos yeux les apports les plus originaux de la linguistique des discours oraux aux théories contemporaines du récit.

\section{N O TES}

1. Pour un bref historique de cette question, voir Vincent et Bres (2001).

2. Fayol a bien mis en évidence ce parallélisme: «Les résultats obtenus par Labov et Waletzky tendent donc à confirmer la thèse déjà soutenue par les analystes littéraires; thèse selon laquelle le récit comporterait une organisation interne bien spécifique. Celle-ci serait inhérente au texte lui-même et concernerait la structure des informations fournies. Il est en effet assez facile de mettre en relation, au moins au niveau des concepts manipulés sous des vocables souvent très différents, les catégories isolées par Barthes, Bremond et Propp avec celles utilisées par Labov" (1985: 26).

3. Recherche financée par le Fonds national suisse de la recherche scientifique (subside ${ }^{\circ} 114-065376$, d'octobre 2001 à septembre 2003 et subside $n^{\circ} 101311-101605$, d'octobre 2003 à septembre 2004). Pour une présentation détaillée, voir Bronckart et Groupe LAF (2004).

4. À la suite des travaux de Gülich et Quasthoff (1986) notamment, il convient selon nous de distinguer les récits - qui mettent en place une tension et satisfont au principe de la mise en intrigue - et les relations qui ne présentent pas une telle organisation des événements rapportés. De ce point de vue, le terme de narration renvoie pour nous à une catégorie générale, indépendante des modalités particulières par lesquelles les événements sont récapitulés (voir Filliettaz, 2001).

5. "There are, then, a variety of devices and combinations of devices by which a triggered story can be appropriately introduced. The observable relationship between a story and prior turn-by-turn talk is a product of methodic displays, fitted to the talk so far and to the story to be told. Further, it appears that the particular circumstances under which a story is entered can have consequences for the structure of the actually told story, which itself is fitted to the manner of its introduction" (Jefferson, 1978: 224).

6. Les chiffres correspondent à la numérotation des huit narrations. Voici explicitées les conventions de transcription:

\begin{tabular}{|c|c|}
\hline ...... & pauses de durée variable \\
\hline : & allongements syllabiques \\
\hline . & bribes \\
\hline$\uparrow$ & intonation montante \\
\hline$\downarrow$ & intonation descendante \\
\hline souligné & chevauchements de la parole \\
\hline$[\mathrm{mm}]$ & régulateurs verbaux énoncés par l'interlocuteur \\
\hline MAJuscule & segments accentués \\
\hline XXX & segments ininterprétables \\
\hline
\end{tabular}

7. Pour être en mesure d'étudier finement la manière dont la narration réorganise la temporalité des événements représentés, il importe de recourir à un système de transcription spécifique, par lequel les productions langagières sont segmentées en unités élémentaires. Nous fondons pour notre part cette segmentation sur le concept de clause tel que défini par Berrendonner (2002). Pour cet auteur, la clause ne se confond pas avec les notions de phrase ou de proposition couramment admises par les grammaires traditionnelles. Elle constitue une unité de rection dotée d'une connexité interne et d'une autonomie externe. Cela signifie que les éléments qui la composent (morphèmes ou syntagmes) sont reliés par des rapports de rection (ou d'implication) alors qu'ils n'entretiennent aucun lien du même genre avec les éléments voisins. Dans les transcriptions qui suivent, chaque numéro de ligne renvoie à une clause distincte.

8. En fait, la clause 9 ("là je vois tout de suite que ça marche pas») pourrait être interprétée aussi bien comme narrative (à ce moment précis j'ai vu que ça marchait pas) que comme un commentaire (en général je vois tout de suite que ça marche pas). En considérant le co-texte immédiat dans lequel cette clause intervient, nous privilégions pour notre part une interprétation commentative.

9. Sur cette question des rapports entre narrations et identité, on consultera notamment Vincent $(1994,1996)$ et Filliettaz (2001). 


\section{RÉFÉREN CES BIBLIO G RAPHIQ U ES}

ADAM, J.-M. [1992]: Les Textes. Types et prototypes, Paris, Nathan. BerRendonNer, A. [2002]: "Les deux syntaxes ", Verbum, XXIV, 23-35. BONU, B. [2001] : «Les évaluations conversationnelles dans la narration ", Revue québécoise de linguistique, vol. 29, n 1, 51-69. BRES, J. [1994a]: La Narrativité, Louvain-la-Neuve, Duculot;

(dir.) [1994b] : Le Récit oral suivi de Questions de narrativité, Montpellier, Praxiling;

[2001]: «De la textualité narrative en récit oral: l'enchaînement des propositions", Revue québécoise de linguistique, vol. 29, n 1, 23-49. BRONCKART, J.-P. et Groupe LAF (dir.) [2004] : Agir et discours en situation de travail, Genève, Cahiers de la section des sciences de l'éducation, no 103 , Université de Genève.

Charaudeau, P. [1994]: "L'acte narratif dans l'interlocution ", dans J. Bres (dir.), 23-35.

FAYOL, M. [1985]: Le Récit et sa construction, Neuchâtel, Delachaux et Niestlé.

FILLIETTAZ, L. [2001] : «Formes narratives et enjeux praxéologiques: quelques remarques sur les fonctions du raconter en contexte transactionnel ", Revue québécoise de linguistique, vol. 29, n 1, 123-153;

[2002]: La Parole en action. Éléments de pragmatique psychosociale, Québec, Nota bene.

GOFFMAN, E. [(1959) 1973]: La mise en scène de la vie quotidienne, tome 1 (La Présentation de soi), Paris, Minuit.

GÜlich, E. et U.M. QUASTHOFF [1986]: «Story-telling in Conversation.
Cognitive and Interactive Aspects ", Poetics, n 15, 217-241. JEFFERSON, G. [1978] : «Sequential Aspects of Storytelling in Conversation ", dans J. Schenkein (dir.), Studies in the Organization of Conversational Interaction, New York, Academic Press, 219-248. Kerbrat-Orecchioni, C. [1980]: L'Énonciation. De la subjectivité dans le langage, Paris, Armand Colin.

LABOV, W. [(1972) 1978]: «La transformation du vécu à travers la syntaxe narrative", Le Parler ordinaire, Paris, Minuit, 289-355.

LAFOREST, M. (dir.) [1996] : Autour de la narration, Québec, Nuit blanche éditeur.

LAFOREST, M. et D. VINCENT [1996] : «Du récit littéraire à la narration quotidienne", dans M. Laforest (dir.), 13-28.

REVAZ, F. [1997]: Les Textes d'action, Paris, Klincksieck (publications du Centre d'études linguistiques des textes et des discours, Université de Metz);

[2004]: "Qu'est-ce que vous avez fait? Verbalisation de l'agir en situation d'entretien ", Cahiers de la section des sciences de l'éducation, no 103, Université de Genève, 263-312.

SACKS, H. [1992] : Lectures on Conversation, 2 vol., Oxford, Blackwell. VINCENT, D. [1994]: "La fonction des narrations dans les entrevues sociolinguistiques", dans J. Bres (dir.), 37-48; $29-46$.

VinCENT, D. et J. BRes [2001]: "Pratiques du récit oral», Revue québécoise de linguistique, vol. 29, nº 1, 7-10. 